\title{
Flavonoids from Triticum aestivum inhibit adipogenesis in 3T3-L1 cells by upregulating the insig pathway
}

\author{
BARUN POUDEL ${ }^{1,2^{*}}$, SARMILA NEPALI $^{2 *}$, MINGJIE XIN $^{1}$, HYEON-HUI KI $^{2}$, \\ YOUNG-HO KIM ${ }^{3}$, DAE-KI KIM ${ }^{2}$ and YOUNG-MI LEE ${ }^{1}$
}

${ }^{1}$ Department of Oriental Pharmacy, College of Pharmacy and Wonkwang-Oriental Medicines Research Institute, Wonkwang University, Iksan, Jeonbuk 570-749; ${ }^{2}$ Department of Immunology and Institute of Medical Sciences, Medical School, Chonbuk National University, Jeonju, Jeonbuk 561-756; ${ }^{3}$ Department of Pharmacognosy,

College of Pharmacy, Chungnam National University, Daejeon 305-764, Republic of Korea

Received May 30, 2014; Accepted February 13, 2015

DOI: $10.3892 / \mathrm{mmr} .2015 .3700$

\begin{abstract}
The present study aimed to compare the potential anti-adipogenic effects and underlying mechanisms of the luteolin, isoscoparin and isoorientin flavonoids, purified from Triticum aestivum sprout (TA) in 3T3-L1 cells. The cells were treated with different concentrations of flavonoids for 8 days and the lipid accumulation was assessed using Oil-Red-O staining. The expression levels of the transcription factors and the genes involved in adipogenesis in the cells were assessed by reverse transcription-quantitative polymerase chain reaction and western blotting. The results demonstrated that $10 \mu \mathrm{M}$ luteolin, isoscoparin or isoorientin inhibited lipid deposition in the cells by 74,63 and $65 \%$, respectively. The flavonoids also significantly inhibited the transcriptional regulators of adipogenesis, including peroxisome proliferator-activated receptor- $\gamma$, CAAT/enhancer binding protein- $\alpha$ and sterol regulatory element binding protein (SREBP)-1c, compared with the control cells. Similarly, there was a significant downregulation of the adipocyte specific markers associated with lipid metabolism, including activating protein-2, fatty acid synthase, hormone-sensitive lipase and lipoprotein lipase, in the flavonoid treated cells. Notably, the cells treated with the
\end{abstract}

Correspondence to: Professor Young-Mi Lee, Department of Oriental Pharmacy, College of Pharmacy and Wonkwang-Oriental Medicines Research Institute, Wonkwang University, 460 Iksandae-ro, Iksan, Jeonbuk 570-749, Republic of Korea

E-mail:ymlee@wku.ac.kr

Professor Dae-Ki Kim, Department of Immunology and Institute of Medical Sciences, Medical School, Chonbuk National University, Deokjin-dong, Deokjin-gu, Jeonju, Jeonbuk 561-756, Republic of Korea

E-mail:daekim@jbnu.ac.kr

*Contributed equally

Key words: Triticum aestivum, extract, flavonoids, 3T3-L1 cells, anti-adipogenesis flavonoids demonstrated increased expression levels of the insulin-induced genes, insig-1 and insig-2, which may have inhibited the activation of the adipogenic transcription factor, SREBP, eventually leading to the inhibition of adipogenesis. Taken together, these results revealed that the flavonoids from TA possessed an inhibitory effect on adipogenesis through downregulation of adipogenic transcription factors and genes associated with lipid metabolism, and the upregulation of insig 1 and 2, suggesting that the flavonoids from TA may be potential therapeutic agents for the prevention and treatment of obesity.

\section{Introduction}

Obesity is a major risk factor of metabolic disorders, including type 2 diabetes, hypertension, hyperlipidemia and arteriosclerosis. The development of obesity is characterized by an increase in adipose tissue cell number (hyperplasia) and cell size (hypertrophy) (1). Genetic, metabolic and nutritional factors are crucial in the development of obesity (2). Therefore, understanding the nutrients that affect adipocyte differentiation may assist in reducing the healthcare burden associated with obesity (3).

Adipogenesis is the process by which fibroblastic preadipocytes are converted into fat laden adipocytes. The 3T3-L1 cell line is considered an optimal in vitro model to investigate adipogenesis, as it exhibits a high potential to differentiate from a preadipocyte to an adipocyte and also exhibits morphological and biochemical properties similar to the development of obesity in humans (4). Adipogenesis involves the stimulation of a series of transcriptional factors, including peroxisome proliferator-activated receptor- $\gamma$ (PPAR $\gamma)$, CAAT/enhancer binding protein- $\alpha(\mathrm{C} / \mathrm{EBP} \alpha)$ and sterol regulatory element binding proteins (SREBPs) (5). Among these, PPAR $\gamma$, which is also activated by SREBP, is considered a key transcription factor for adipogenesis, as it stimulates the expression levels of several genes, which are crucial for adipogenic processes, including fatty acid synthase (FAS), fatty acid binding proteins (aP2), hormone-sensitive lipase (HSL) and lipoprotein lipase (LPL) $(1,6,7)$. It has been revealed that the insulin-induced gene (insig) protein family of endoplasmic reticulum, insig-1 
and insig-2, are important in cholesterol metabolism (8) and it has been reported that rats fed a high fat-diet exhibit increased expression of insig-1 in white adipose tissue (7). Insig-1 binds to SREBP cleavage activating protein and prevents the proteolytic action of SREBP to activate transcription factors and, therefore, prevents its effects on gene transcription (7), which eventually inhibits preadipocyte differentiation and lipogenesis in the adipocytes (8). In addition, insig-2 suppresses the proteolytic action of SREBP, inhibiting the development of adipogenesis (9).

Although several anti-obesity drugs are commercially available, their safety and efficacy is questioned. Therefore, the identification of naturally occurring compounds, which modulate obesity, may offer beneficial therapeutic efficiency (10). Triticum aestivum sprouts (TA), commonly termed wheat, is one of the major crops grown worldwide. During germination/sprouting, a number of useful phenolic compounds develop in the seeds of TA (11). The germinated wheat leaves are consumed as a rich source of soluble fiber, vitamins, antioxidants and minerals (11). Our previous study demonstrated that extract from TA exhibited antihyperlipidemic and antidiabetic effects in streptozotocin-induced diabetic mice $(12,13)$. TA extract has also been observed to suppress lipid accumulation in 3T3-L1 cells (14) and TA inhibits liver lipid accumulation in high fat-fed mice (15). Our previous study demonstrated that, in leptin deficient ob/ob mice, administration of TA extract reduced the levels of blood glucose and cholesterol (16). These anti-obesity-associated effects of the TA extract prompted the purification of the compounds present in the extract. As a result, the leuteolin, isoscoparin and isoorientin flavonoids were present in the extract. Although the flavonoids such as luteolin, isoscoparin and isoorientin are present in TA $(17,18)$, their effects in the regulation of adipogenesis remain to be elucidated.

The present study was performed to compare the anti-adipogenic effects of the luteolin, isoscoparin and isoorientin flavonoids, purified from TA, in 3T3-L1 cells. Furthermore, the study aimed to elucidate the underlying mechanisms associated with their anti-adipogeneic effects.

\section{Materials and methods}

Materials and reagents. Dexamethasone (Dex), insulin, 3-isobutyl-1-methylxanthine(IBMX), RNase AandOil-Red-O (ORO) were purchased from Sigma-Aldrich (St. Louis, MO, USA). Dulbecco's modified Eagle's medium (DMEM) and fetal calf serum (FCS) were purchased from HyClone, (Logan, UT, USA). TRIzol reagent and the SuperScript III kit were obtained from Invitrogen Life Technologies (Carlsbad, CA, USA). The Cell Counting Kit-8 (CCK-8) was purchased from Dojindo Molecular Technologies (Rockville, MD, USA). Rabbit anti-PPAR $\gamma$ (cat. no. C26H12) and rabbit anti-FAS (cat. no. C20G5) monoclonal antibodies were purchased from Cell Signaling Technology, Inc. (Beverly, MA, USA). Rabbit polyclonal anti-CEBP $\alpha$ antibody (cat. no. sc-61), the protein assay kit, including radioimunoprecipitation (RIPA) buffer, goat anti-rabbit (cat. no. sc-2030) and goat anti-mouse (cat. no. sc-2005) horseradish peroxidase (HRP)-conjugated immunoglobulin G secondary antibodies, and mouse monoclonal anti- $\beta$-actin antibody (cat. no. sc-47778), were obtained from Santa Cruz Biotechnology, Inc. (Dallas, TX, USA).

Extraction and purification of the flavonoids from TA. TA was obtained from the National Institute of Crop Science (Jeonbuk, Korea) and was subsequently dried in a freezer dryer. Isoscoparin and isoorientin were purified from TA as reported previously (17). Briefly, dried TA was extracted with $\mathrm{MeOH}$ (DC Chemical Co., Ltd., Seoul, Korea). The MeOH extracts were then partitioned in turn using four solvents, n-hexane (DC Chemical Co., Ltd.), $\mathrm{CH}_{2} \mathrm{CL}_{2}$, EtOAc and n-BuOH (Junsei Chemical Co., Ltd., Tokyo, Japan). The EtOAc fraction of the plant was subjected to column chromatography using silica gel (70-230, 230-400 mesh; Merck, Whitehouse Station, NJ, USA) with $\mathrm{CH}_{2} \mathrm{CL}_{2}-\mathrm{MeOH}$ (5:1) as a solvent to yield concentrated fractions. In secondary column chromatography using YMC RP-18 resins (75 $\mu$ m, Fuji Silysia Chemical Ltd., Kasugai, Japan) as absorbent, isoscoparin was purified with $\mathrm{MeOH}_{2} \mathrm{O}$ (2:3) as eluent, and then isoorientin was eluted with acetone (DC Chemical Co., Ltd.) $\mathrm{H}_{2} \mathrm{O}$ (1:3). Luteolin was obtained from Sigma-Aldrich.

Cell culture and adipocyte differentiation assay. The 3T3-L1 preadipocytes were obtained from American Type Culture Collection (Rockville, VA, USA) and maintained in DMEM, supplemented with $10 \%$ FCS and $1 \% 100 \mathrm{U} / \mathrm{ml}$ penicillin and $100 \mathrm{U} / \mathrm{ml}$ streptomycin (Welgene, Daegu, Korea). incubated at $37^{\circ} \mathrm{C}$ in a $5 \% \mathrm{CO}_{2}$ humidified incubator. The preadipocytes were induced to differentiate 2 days post-confluence (day 0 ) by adding $0.5 \mathrm{mM}$ IBMX, $1 \mu \mathrm{M}$ Dex and $10 \mu \mathrm{g} / \mathrm{ml}$ insulin (MDI) for 2 days at $37^{\circ} \mathrm{C}$ in a $5 \% \mathrm{CO}_{2}$ humidified incubator (19). The culture medium was subsequently changed to DMEM/10\% FCS containing insulin $(10 \mu \mathrm{g} / \mathrm{ml})$. Following incubation for 2 days, the medium was replaced with DMEM/10\% FCS and incubated for another 2 days at $37^{\circ} \mathrm{C}$ in a $5 \% \mathrm{CO}_{2}$ humidified incubator. The individual flavonoids $(0,1,5$, or $10 \mu \mathrm{M})$ were added on day 0 during differentiation, until the cells were harvested for the subsequent experiments.

Cytotoxicity assay. The cells were treated with MDI and various concentrations of the flavonoids for 2,4 or 8 days at $37^{\circ} \mathrm{C}$ in a $5 \% \mathrm{CO}_{2}$ humidified incubator, and cell viability was measured using a CCK-8 kit, according to the manufacturer's instructions. The absorbance was measured at $450 \mathrm{~nm}$ on a microplate reader (Biochrom Anthos Zenyth 200; Biochrom Ltd., Cambridge, UK).

Oil-red staining. The cells were differentiated, as described above. On day 8, the cells were stained with Oil-Red-O (ORO), according to the manufacturer's instructions to visualize the lipid accumulation in the cells. The intracellular lipid content was measured by extracting ORO using $100 \%$ isopropanol (Merck KGaA, Darmstadt, Germany) and the absorbance at $520 \mathrm{~nm}$ was recorded using a spectrophotometer (Optizen 2120 UV; Mecasys Co., Ltd., Daejeon, Korea).

Reverse transcription-quantitative polymerase chain reaction (RT-qPCR). The total RNA was extracted using TRIzol reagent, according to the manufacturer's instructions. RNA $(2 \mu \mathrm{g})$ was used for cDNA synthesis using the Super Script ${ }^{\mathrm{TM}}$ III kit. The 
Table I. Primers used for reverse transcription-quantitative polymerase chain reaction.

\begin{tabular}{|c|c|c|c|}
\hline Primer & Accession no. & Sequence $\left(5^{\prime}-3^{\prime}\right)$ & Base pairs \\
\hline GAPDH & NM_001289726 & $\begin{array}{l}\text { Forward CATGGCCTTCCGTGTTC } \\
\text { Reverse CCTGGTCCTCAGTGTAGC }\end{array}$ & 152 \\
\hline PPAR $-\gamma^{2}$ & XM_006505744.1 & $\begin{array}{l}\text { Forward GATGGAAGACCACTCGCATT } \\
\text { Reverse AACCATTGGGTCAGCTCTTG }\end{array}$ & 115 \\
\hline $\mathrm{C} / \mathrm{EBP} \alpha$ & NM_001287514 & $\begin{array}{l}\text { Forward TTGTTTGGATTTATCTCGGC } \\
\text { Reverse CCAAGAAGTCGGTGGACAAG }\end{array}$ & 95 \\
\hline FAS & NM_007988.3 & $\begin{array}{l}\text { Forward TGCTCCCAGCTGCAGGC } \\
\text { Reverse GCCCGGTAGCTCTGGGTGTA }\end{array}$ & 107 \\
\hline $\mathrm{aP} 2$ & XM_006530048 & $\begin{array}{l}\text { Forward AGCCTTTCTCACCTGGAAGA } \\
\text { Reverse TTGTGGCAAAGCCCACTC }\end{array}$ & 136 \\
\hline LPL & XM_006509563 & $\begin{array}{l}\text { Forward GGACGGTAACGGGAATGTATGA } \\
\text { Reverse TGACATTGGAGTCAGGTTCTCTGT }\end{array}$ & 81 \\
\hline HSL & XM_006539572 & $\begin{array}{l}\text { Forward GGAGCACTACAAACGCAACGA } \\
\text { Reverse TCGGCCACCGGTAAAGAG }\end{array}$ & 67 \\
\hline SREBP-1c & XM_006532713 & $\begin{array}{l}\text { Forward GGTTTTGAACGACATCGAAGA } \\
\text { Reverse CGGGAAGTCACTGTCTTGGT }\end{array}$ & 61 \\
\hline Insig-1 & NM_153526 & $\begin{array}{l}\text { Forward TGTGGTTCTCCCAGGTGACT } \\
\text { Reverse TAGCCACCATCTTCTCCTCC }\end{array}$ & 109 \\
\hline Insig-2 & NM_133748 & $\begin{array}{l}\text { Forward TGAAGCAGACCAATGTTTCAA } \\
\text { Reverse GGTGAACTGGGGGTCTCC }\end{array}$ & 90 \\
\hline
\end{tabular}

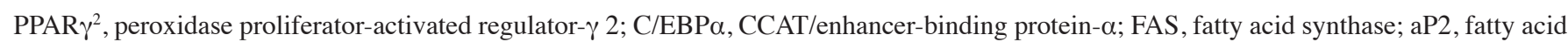
activating protein 2; LPL, lipoprotein lipase; HSL, hormone-sensitive lipase; SREBP-1c, sterol regulatory element binding protein 1c; insig1, insulin-induced gene 1 ; insig 2 insulin-induced gene 2 .

mRNA expression levels were quantitatively determined using an ABI Real-Time PCR system (Applied Biosystems, Inc., Foster City, CA, USA) and a SYBR Green PCR Master mix (Life Technologies, Carlsbad, CA, USA). GAPDH was used as the internal control. The specific primers were obtained from Genotech (Daejeon, Korea), and are listed in Table I. qPCR was performed using the following cycle conditions: $95^{\circ} \mathrm{C}$ for $10 \mathrm{~min}$, followed by 40 cycles at $95^{\circ} \mathrm{C}$ for $15 \mathrm{sec}$ and $60^{\circ} \mathrm{C}$ for $1 \mathrm{~min}$. Every experiment was carried out in triplicate. The results are expressed as the relative expression levels compared with the control.

Western blot analysis. Western blot analysis was performed, as previously described (20). The cells were lysed in ice-cold RIPA buffer for $40 \mathrm{~min}$ and centrifuged $(12,000 \mathrm{x} \mathrm{g})$ for $20 \mathrm{~min}$ at $4^{\circ} \mathrm{C}$. The protein concentration was measured using a bicinchoninic acid assay (Sigma-Aldrich). The lysates $(30 \mu \mathrm{g})$ were separated on $8 \%$ sodium dodecyl sulfate-polyacrylamide gel electrophoresis gels and transferred onto polyvinylidene difluoride membranes (Amersham Pharmacia Biotech Co., Ltd., Piscataway, NJ, USA). The membranes were subsequently blocked with 5\% non-fat milk in tris-buffered saline (Amresco LLC, Solon, OH, USA), containing 0.1\% Tween-20 (Sigma-Aldrich) (TBST) for $1 \mathrm{~h}$ at room temperature, and the membranes were probed with the indicated primary antibodies (1:1,000 dilutions) at $4^{\circ} \mathrm{C}$ overnight. The membranes were washed with TBST four times and were subsequently incubated with HRP-conjugated secondary antibodies (1:5,000 dilutions) for $45 \mathrm{~min}$ at room temperature. The membranes were washed with TBST three times, and the proteins were visualized using an enhanced chemiluminescence detection kit (Millipore, Billerica, MA, USA).

Statistical analysis. All data are expressed as the mean \pm standard error of the mean. Statistical significance was determined using Student's t-test. Statistical analysis was performed using Microsoft Excel version 2007 (Microscoft Korea, Seoul, Korea). $\mathrm{P}<0.05$ was considered to indicate a statistically significant difference.

\section{Results}

Flavonoids from TA inhibit lipid accumulation without significant cytotoxicity in 3T3-L1 cells. The 3T3-L1 cells were treated with various concentrations $(0,1,5$ and $10 \mu \mathrm{M})$ of the purified flavonoids for 8 days. On the $2^{\text {nd }}, 4^{\text {th }}$ and $8^{\text {th }}$ days of treatment, the cell viability was determined using a CCK-8 assay. The results demonstrated that the flavonoids caused no significant cytotoxicity towards the differentiating preadipocytes (1-10 $\mu \mathrm{M}$; Fig. 1A-C). This result ruled out the possibility that the anti-adipogenic effects of the flavonoids resulted from cytotoxicity to the cells. ORO staining positively correlates with the amount of lipid stored inside the cells, and has been widely utilized to demonstrate the potential anti-obesity 

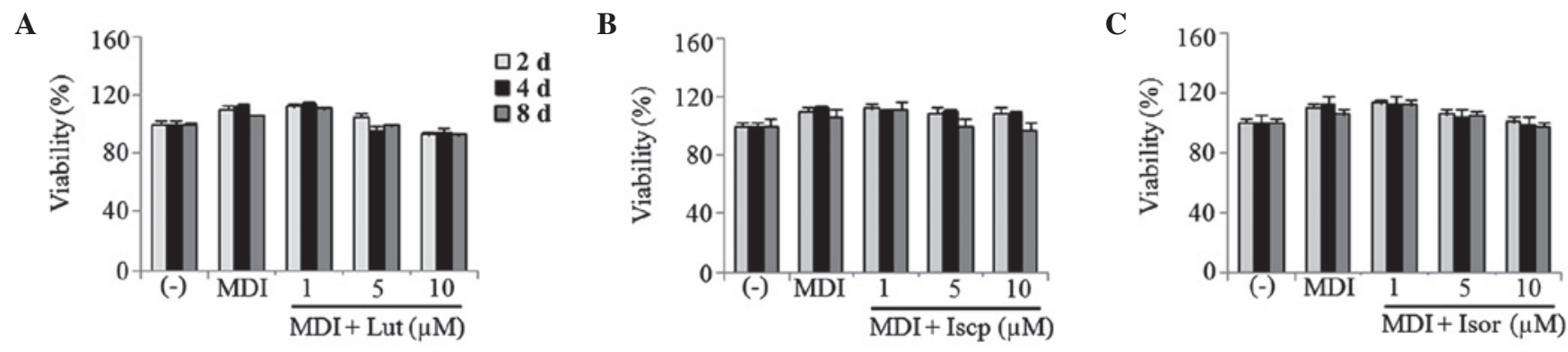

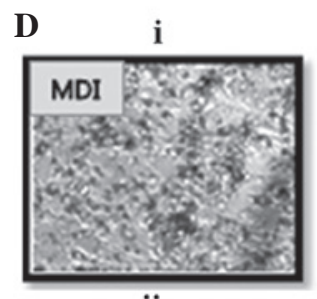

ii
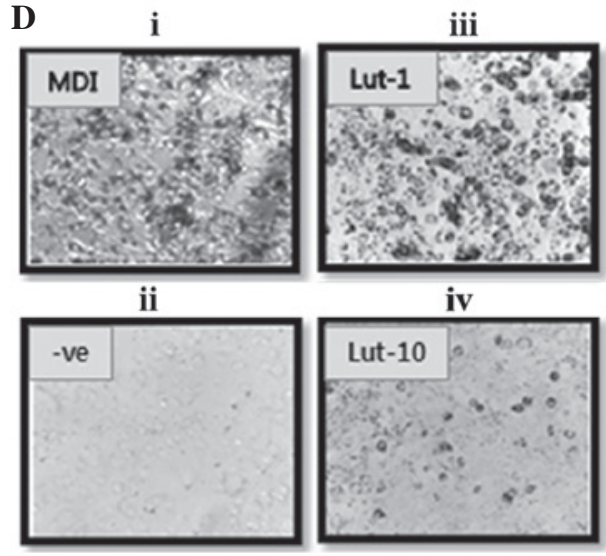

iv

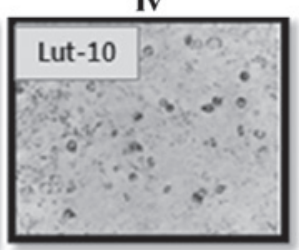

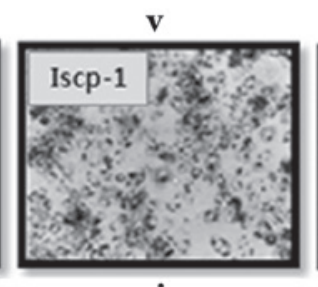

vi

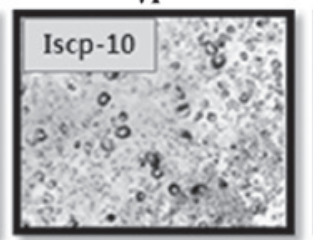

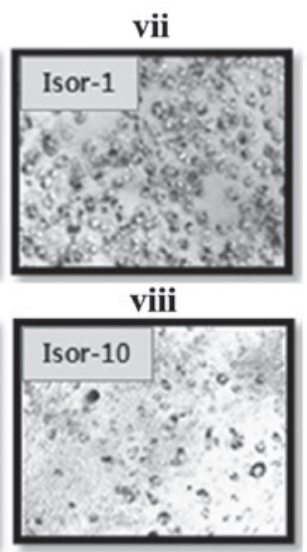

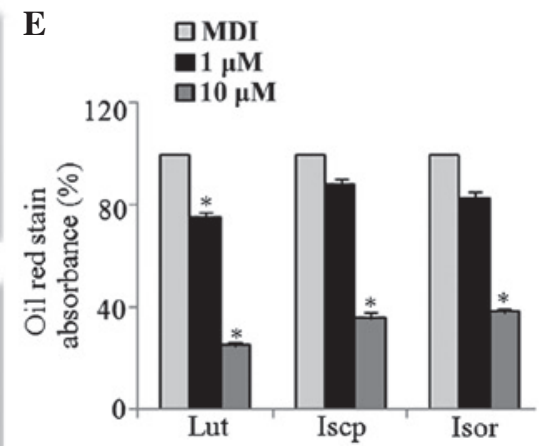

Figure 1. Effect of flavonoids from TA on the cell viability and lipid accumulation in 3T3-L1 cells. The viability of the 3T3-L1 cells treated with (A) Lut (B) Iscp and (C) Isor was determined on the $2^{\text {nd }}, 4^{\text {th }}$ and $8^{\text {th }}$ day of differentiation using a CCK- 8 assay. (D) Images of the microscopic visualization of the ORO staining in the cells on $8^{\text {th }}$ day. (i) MDI-induced adipocytes, (ii) non-induced, MDI-induced and treatment with Lut at (iii) $1 \mu \mathrm{M}$ and (iv) $10 \mu \mathrm{M}$; Iscp at (v) $1 \mu \mathrm{M}$ and (vi) $10 \mu \mathrm{M}$; Isor at (vii) $1 \mu \mathrm{M}$ and (viii) $10 \mu \mathrm{M}$ (magnification, $\mathrm{x} 40$ ). (E) ORO-stained lipids were extracted using isopropanol, and the absorbance was measured using a spectrophotometer at $520 \mathrm{~nm}$. The data are expressed as the mean \pm standard error of the mean ( $\mathrm{n}=3$; ${ }^{*} \mathrm{P}<0.05$, compared with the control). TA, Triticum aestivum; Lut, luteolin; Iscp, isoscoparin; Isor, isoorienin; CCK, cell counting kit; MDI, insulin; ORO, oil red-O.

effects of natural products. Therefore, to visualize the lipid accumulation in the cytoplasm during adipogenesis, the cells were treated with the indicated concentrations of the flavonoids during differentiation and were analyzed using ORO staining. As shown in Fig. 1D, lipid accumulation was inhibited dose-dependently by all three flavonoids. At $10 \mu \mathrm{M}$, the inhibitory rates were 75, 64 and $65 \%$ following treatment with luteolin, isoscoparin and isoorientin, respectively (Fig. 1E).

Effects of the flavonoids from TA on the expression of adipogenic transcription factors in the 3T3-L1 cells. To examine the effect of the flavonoids on the expression levels of the predominant adipogenic transcription factors in the 3T3-L1 cells during adipogenesis, the cells were treated with or without the flavonoids $(0,1$ and $10 \mu \mathrm{M})$. After 2 days, the mRNA expression levels of PPAR $\gamma$ and $\mathrm{C} / \mathrm{EBP} \alpha$ were measured and the inhibition of lipid accumulation was found to be associated with the downregulation of the transcription factors in a dose-dependent manner, compared with the untreated control cells (Fig. 2A and B). Furthermore, the protein expression levels of these factors were determined on the $4^{\text {th }}$ day following treatment with the flavonoids. At $10 \mu \mathrm{M}$, the protein expression levels of PPAR $\gamma$ were suppressed 47,50 and $46 \%$, and those of $\mathrm{C} / \mathrm{EBP} \alpha$ were suppressed 66,58 and $76 \%$, by luteolin, isoscoparin and isoorientin, respectively, compared with the controls (Fig. 2C-F).

Effects of the flavonoids from TA on the expression levels of adipogenesis-associated genes in the 3T3-L1 cells. Since the adipogenic transcription factors were downregulated by the flavonoids, the expression levels of adipocyte-specific genes involved in fatty acid synthesis, FAS and aP2, and lipogenic genes, LPL and HSL, were also quantified. Significant inhibition of the mRNA and protein expression levels of FAS was demonstrated following treatment with the flavonoids. At $10 \mu \mathrm{M}$, the protein expression of FAS was suppressed by 42,68 and $33 \%$, by luteolin, isoscoparin and isoorientin, respectively compared with the control (Fig. 3A-C). Other adipocyte-specific genes, including aP2, HSL and LPL, were also inhibited in a dose-dependent manner compared with the control (Fig. 3D-F).

Effects of the flavonoids from TA on the expression of insig-1 and insig-2 in the 3T3-L1 cells. As insig-1 and 2 are known to inhibit the activation of SREBP-1c and inhibit adipogenesis (7), the present study aimed to examine whether the flavonoids from TA modulate the expression of these genes. For this, RT-qPCR was performed to assess their levels of expression in the cells treated with, or without, each flavonoid. The results indicated that the flavonoids inhibited the expression of SREBP1c (Fig. 4A). Furthermore, the expression levels of insig- 1 and 2 were upregulated by the flavonoids in the 3T3-E1 cells compared with the control (Fig. 4B-C).

\section{Discussion}

Obesity is a major public health concern, which arises due to an imbalance in energy intake and energy expenditure. 
A

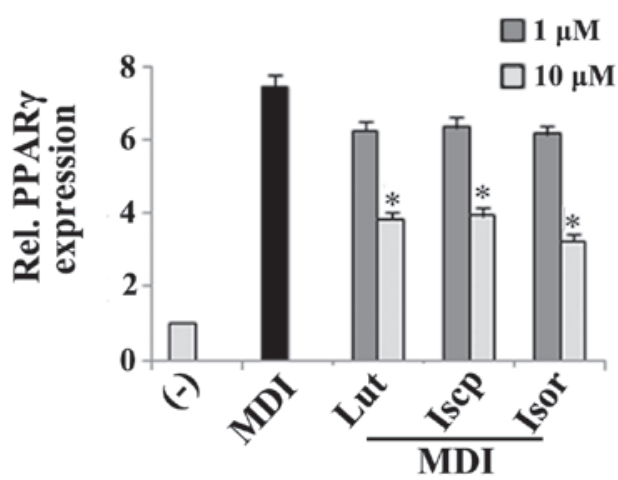

C

E

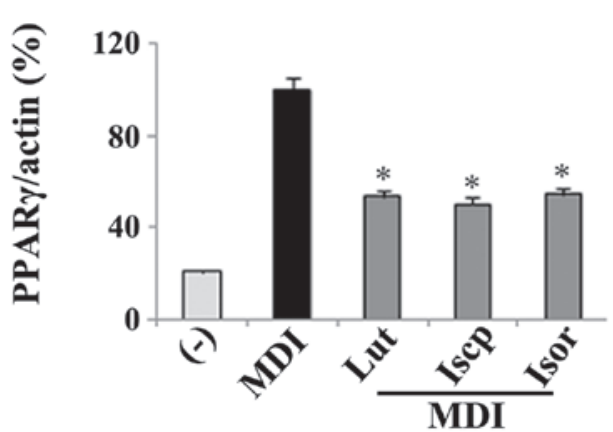

B

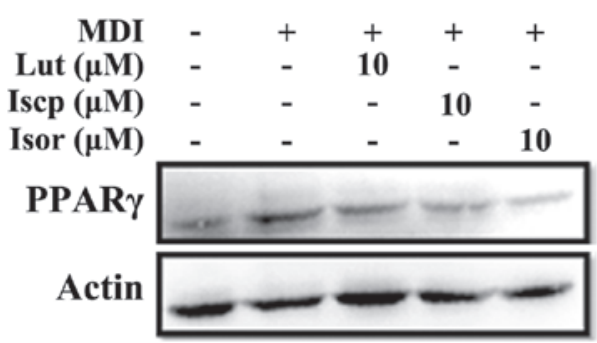

D

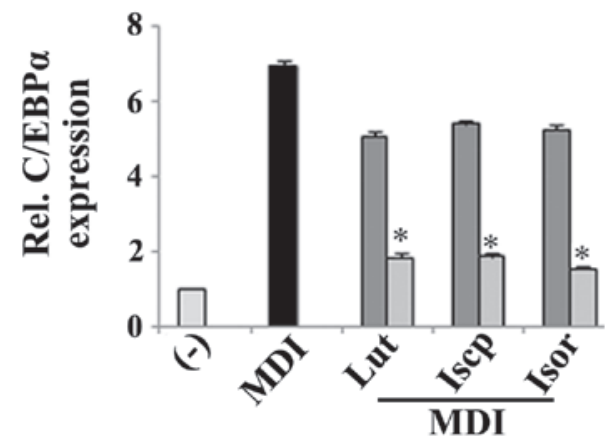

F

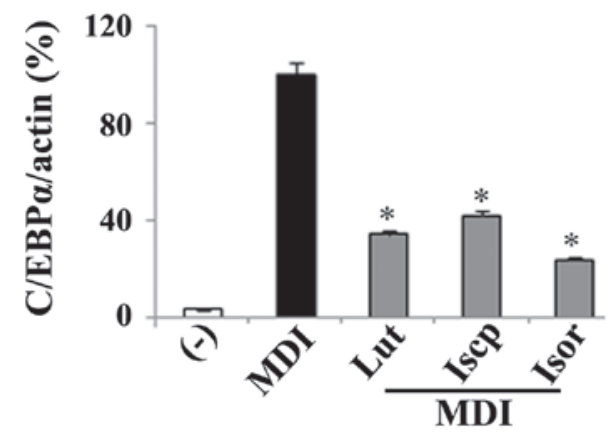

Figure 2. Effects of flavanoids from TA on the expression levels of adipogenic transcriptional factors in 3T3-L1 adipocytes. The 3T3-L1 cells were incubated with or without the Lut, Iscp or Isor flavonoids, in the presence or absence of MDI, at the indicated doses. The analysis of the mRNA expression levels was performed by reverse transcription-quantitative polymerase chain reaction following 2 days treatment. The protein expression levels were analyzed following 4 days treatment. The effect of the flavanoids from TA on the (A) mRNA and (B) protein expression levels of PPAR $\gamma$ and the (C) percentage ratio (PPAR $\gamma /$ actin) of the band intensity were determined. The (D) mRNA and (E) protein expression levels of C/EBP $\alpha$, and the (F) ratio percentage (C/EBP $\alpha /$ actin) of the band intensity were determined. The data are expressed as the mean \pm standard error of the mean ( $\mathrm{n}=3$; ${ }^{*} \mathrm{P}<0.05$, compared with the control). TA, Triticum aestivum; Lut, luteolin; Iscp, isoscoparin; Isor, isoorienin; MDI, insulin; PPAR, peroxisome proliferator-activated receptor; C/EBP, CAAT/enhancer binding protein; Rel, relative.

Consequently, this imbalance leads to the pathological growth of adipocytes. Although there are medications to induce weight loss, they are associated with negative side effects. For example, orlistat is known to cause fecal fat loss and gastrointestinal symptoms (19). Therefore, it is necessary to focus investigations on healthy foods and novel drugs, which are safe and effective, for the prevention and treatment of obesity $(10,21)$.

Adiposity is associated with an increase in the number of adipocytes and the lipid content of adipocytes. The results from the present study demonstrated that the flavonoids $(1-10 \mu \mathrm{M})$ purified from TA had no effect on the viability of differentiating 3T3-L1 cells. This result suggested that flavonoids-induced cytotoxicity did not account for the inhibition of adipogenesis. Other experiments demonstrated that the flavonoids significantly reduced lipid accumulation in the 3T3-L1 cells. The regulation of differentiation of preadipocytes into adipocytes is mediated by several transcription factors. Among these, PPAR $\gamma, \mathrm{C} / \mathrm{EBP}$ s and SREBP1c are known to be critical during the differentiation of the cells (7). During the early adipocyte differentiation stages, activation of $\mathrm{C} / \mathrm{EBP} \beta$ and $\delta$ is known to activate $\mathrm{C} / \mathrm{EBP} \alpha$ and $\mathrm{PPAR} \gamma$, which control adipogenesis and insulin sensitivity in adipocytes (22). Therefore, the present study analyzed the expression profiles of adipocyte genes that are involved in the inhibition of adipogenesis, following treatment with the flavonoids. Treatment with the flavonoids during the early stages of differentiation caused a significant inhibition of the expression levels of $\mathrm{C} / \mathrm{EBP} \alpha$ and PPAR $\gamma$. Their activation is known to regulate the adipocyte differentiation markers and genes associated with lipid metabolism, including aP2, FAS, LPL, and HSL $(21,23)$.

FAS is a lipogenic enzyme, which facilitates the synthesis and cytoplasmic storage of large triglycerides (24). It is associated with lipid accumulation during adipogenesis (25). aP2 is 

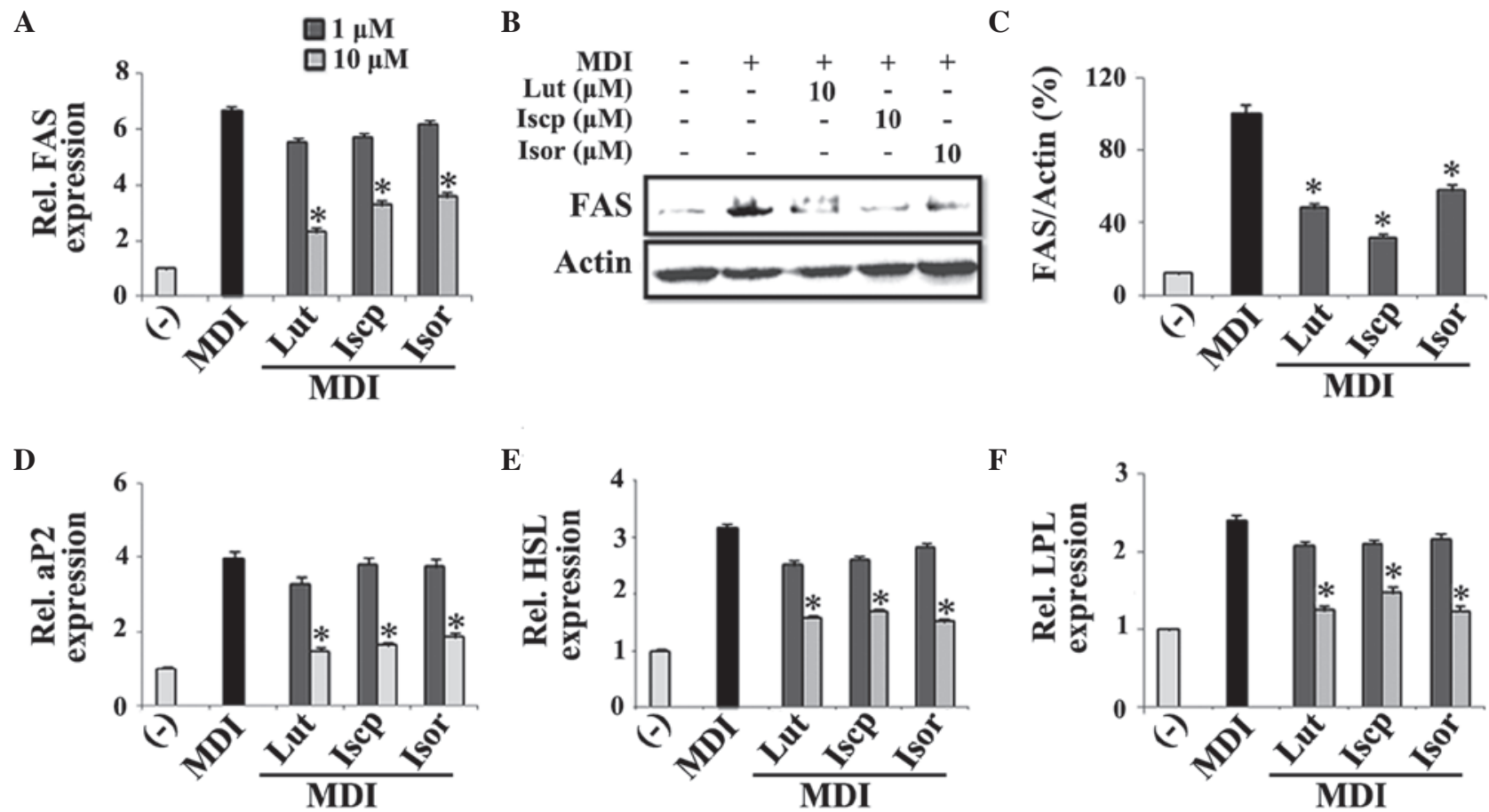

Figure 3. Effects of the flavanoids from TA on the expression levels of adipocyte differentiation markers in 3T3-L1 adipocytes. The 3T3-L1 cells were treated with differentiating medium in the presence or absence of flavonoids for 2 or 4 days for determining the mRNA and protein expression levels, respectively. The effects of the flavonoids from TA on the (A) mRNA and (B) protein expression levels of FAS, the (C) percentage ratio (FAS/actin), and the mRNA expression levels of (D) aP2, (E) HSL and (F) LPL were assessed. The data are expressed as the mean \pm standard error of the mean ( $=3$; ${ }^{*} \mathrm{P}<0.05$, compared with the control). TA, Triticum aestivum; Lut, luteolin; Iscp, isoscoparin; Isor, isoorienin; MDI, insulin; FAS, fatty acid synthase; aP2, fatty acid binding protein; HSL, hormone-sensitive lipase; LPL, lipoprotein lipase; Rel, relative.

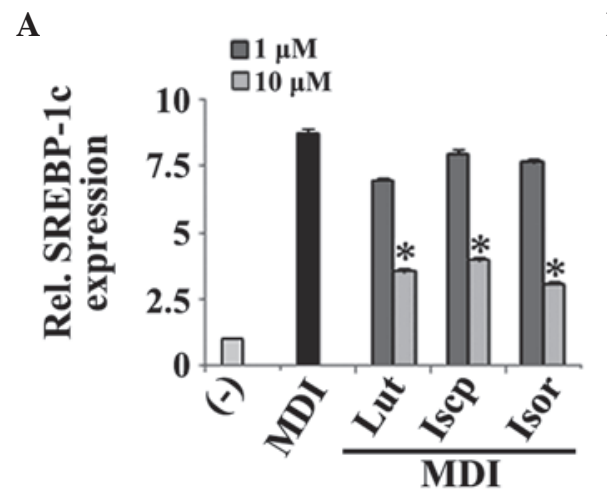

B

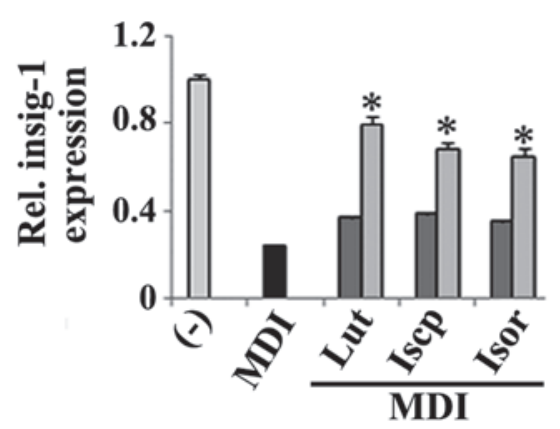

C

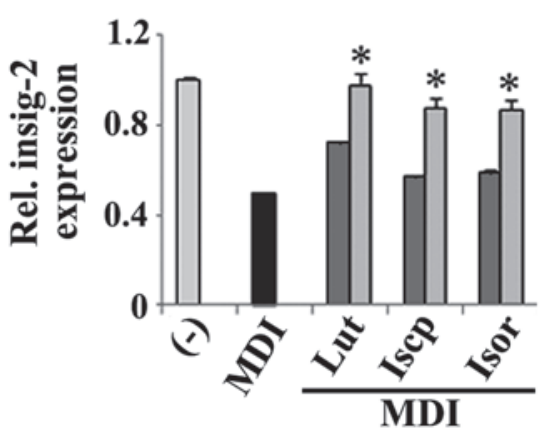

Figure 4. Effects of the flavanoids from TA on the expression levels of the genes involved in the insig pathway in 3T3-L1 cells. The 3T3-L1 cells were treated with differentiating medium in the presence or absence of the flavonoids for 2 days. The mRNA expression levels of (A) SREBP-1c, (B) insig-1 and (C) insig-2 were determined. The data are expressed as the mean \pm standard error of the mean $\left(\mathrm{n}=3 ;{ }^{*} \mathrm{P}<0.05\right.$, compared with the control). TA, Triticum aestivum; Lut, luteolin; Iscp, isoscoparin; Isor, isoorienin; MDI, insulin; SREBP, sterol regulatory element binding protein; Insig, insulin-induced gene; Rel, relative.

the terminal differentiation marker of adipocyte differentiation, which causes the uptake of cellular long-chain fatty acids during fatty acid metabolism and obesity (26). Therefore, the present study examined the effect of the flavonoids on the regulation of the expression levels of FAS and aP2 in 3T3-L1 cells during adipogenesis. These genes were significantly suppressed in a dose-dependent manner, suggesting that the downregulation of FAS and aP2 may have resulted from the decreased utilization of fatty acid transport in the 3T3-L1 cells. HSL is a key lipolytic response gene associated with lipid catabolism in adipocytes, as it hydrolyzes triacylglycerol to monoacylglycerol and free fatty acid (22). LPL is a marker of early adipocyte differentiation, and its overexpression initiates lipid accumulation during adipogenesis $(27,28)$. In the present study, the expression levels of HSL and LPL were inhibited by the flavonoids in a dose-dependent manner, which suggested that the anti-adipogenic effects of the flavonoids were mediated via the downregulation of the adipogenic process. Consistent with these results, several previous studies have reported anti-adipogenic effects of compounds derived from medicinal plants by inhibiting the expression levels of $\mathrm{C} / \mathrm{EBP} \alpha$, PPAR $\gamma$ and the genes involved in the adipogenic process. For example, ursolic acid and resveratrol-amplified grape skin extracts inhibit the adipogenesis of 3T3-L1 cells by 
downregulating the expression levels of the PPAR $\gamma$ and CEBP isoforms $(28,29)$.

Furthermore, the present study aimed to identify a signaling pathway, by which the flavonoids modulate adipogenesis in 3T3-L1 cells. It was hypothesized that the flavonoids may induce a regulator, which suppresses adipogenic gene expression. A previous study demonstrated that the insig proteins are important for downregulation of the SREBP pathway, which regulates cholesterol and fatty acid synthesis (8). Another study demonstrated that insig-1 inhibits lipogenesis and adipocyte differentiation (30). However, insig-2 is found to be highly expressed in adipose tissue and has been suggested to be a crucial factor in adipose metabolism (7). Notably, during adipocyte differentiation, the changes in the expression of insig-2 is higher than that of insig-1 (7). Consistent with these studies, the data from the present study demonstrated that the expression of insig- 2 was higher than insig-1 in the flavonoid-treated cells compared with the control. This suggested that treatment with the flavonoids increased the expression levels of the insig proteins, which may have inhibited the activation of SREBP1c and reduced the expression levels of adipogenic genes in the 3T3-L1 cells.

Taken together, the results of the present study demonstrated that the flavonoids derived from TA were natural anti-adipogenic molecules, which inhibited adipogenic transcription factors and, consequently, the expression levels of genes involved in adipocyte differentiation and lipid metabolism. This potentially occurred through the upregulation of insig-1 and 2. Additional in vivo investigations are required to confirm the anti-obesity effects of the purified flavonoids assessed in the present study.

\section{Acknowledgements}

This study was supported by grants from Wonkwang University (2014).

\section{References}

1. Kanda K, Nishil K, Kadota A, Nishimoto S, Liu MC and Sagahara T: Nobiletin suppresses adipocyte differentiation of 3T3-L1 cells by an insulin and IBMX mixture induction. Biochim Biophys Acta 1820: 461-468, 2012.

2. Kopelman PG: Obesity as a medical problem. Nature 404: 635-643, 2000.

3. Farmer SR: Regulation of PPAR $\gamma$ activity during adipogenesis. Int J Obes 29: 13-16, 2005.

4. Kwon JY, Seo SG, Heo YS, Yue S, Cheng JX, Lee KW and Kim KH: Piceatannol, natural polyphenolic stilbene, inhibits adipogenesis via modulation of mitotic clonal expansion and insulin receptor-dependent insulin signaling in early phase of differentiation. J Biol Chem 287: 11566-11578, 2012.

5. White UA and Stephens JM: Transcriptional factors that promote formation of white adipose tissue. Mol Cell Endocrinol 318 : 10-14, 2010.

6. Park HS, Kim SH, Kim YS, et al: Luteolin inhibits adipogenic differentiation by regulating PPARgamma activation. Biofactors 35: 373-379, 2009 .

7. Ka SO, Kim KA, Kwon KB, Park JW and Park BH: Silibinin attenutates adipogenesis in 3T3-L1 preadipocytes through potential upregulation of the insig pathway. Int J Mol Med 23: 633-637, 2009

8. Yang T, Espenshade PJ, Wright ME, et al: Crucial step in cholesterol homeostasis: sterols promote binding of SCAP to INSIG-1, a membrane protein that facilitates retention of SREBPs in ER. Cell 110: 489-500, 2002.
9. Goldstein JL, DeBose-Boyd RA and Brown MS: Protein sensors for membrane sterols. Cell 124: 35-46, 2006.

10. Montagut G, Blade C, Blay M, et al: Effects of a grapeseed procyanidin extract (GSPE) on insulin resistance. J Nutr Biochem 21: 961-967, 2010.

11. Lee SH, Lim SW, Lee YM, Lee HS and Kim DK: Polysaccharide isolated from Triticum aestivum stimulates insulin release from pancreatic cells via the ATP-sensitive $\mathrm{K}+$ channel. Int J Mol Med 29: 913-919, 2012.

12. Lee SH, Lee YM, Lee HS and Kim DK: Anti-oxidative and anti-hyperglycemia effects of Triticum aestivum wheat sprout water extracts on the streptozotocin-induced diabetic mice. Korean J Pharmacogn 40: 408-414, 2009;

13. Lee SH, Lim SW, Lee YM, Hur JM, Lee HS and Kim DK: Anti-diabetic effects of Triticum aestivum L. water extracts in $\mathrm{db} / \mathrm{db}$ mice as an animal model of diabetes mellitus type II. Korean J Pharmacogn 41: 282-288, 2010.

14. Lee SH, Xin M, Luyen BTT, et al: Inhibitory effect of Triticum aestivum ethanol extract on lipid accumulation in 3T3-L1 preadipocytes. Yakhak Hoeji 55: 478-484, 2011.

15. Lee SH, Lim SW, Lee YM, Lee SH and Kim DK: Inhibitory effects of Triticum aestivum L. extracts on liver lipid accumulation in high fat-fed mice. Korean J Pharmacogn 42: 309-316, 2011.

16. Lee SH, Lim SW, Mihn NV, et al: Administration of Triticum aestivum water extracts reduce the level of blood glucose and cholesterol in leptin deficient ob/ob mice. J Korean Soc Food Sci Nutr 40: 401-408, 2011.

17. Luyen BTT, Tai BH, Thao NP, Cha JY, Lee YM and Kim YH: A new phenolic component from Triticum aestivum sprouts and its effects on LPS-stimulated production of nitric oxide and TNF-alpha in RAW 264.7 cells. Phytother Res 28: 1064-1070, 2014.

18. Wojakowska A, Perkowski J, Goral T and Stobiecki M: Structural characterization of flavanoid glycosides from leaves of wheat (Triticum aextivum L.) using LC/MS/MS profiling of the target compounds. J Mass Spectrom 48: 329-339, 2013.

19. Poudel B, Lim SW, Ki HH, Nepali S, Lee YM and Kim DK: Dioscin inhibits adipogenesis through the AMPK/MAPK pathway in 3T3-L1 cells and modulates fat accumulation in obese mice. Int J Mol Med 34: 1401-1408, 2014.

20. Poudel B, Yoon DS, Lee JH, Lee YM and Kim DK: Collagen I enhances functional activities of human monocyte-derived dendritic cells via discoidin domain receptor 2. Cell Immunol 278: 95-102, 2012

21. Morimoto C, Kameda K, Tsujita T and Okuda H: Relationships between lipolysis I induced by various lipolytic agents and hormone-sensitive lipase in rat fat cells. J Lipid Res 42: 120-127, 2001.

22. Bennett MK, Lopez JM, Sanchez HB and Osborne TF: Sterol regulation of fatty acid synthase promoter. Coordinate feedback regulation of two major lipid pathways. J Biol Chem 270: 25578-25583, 1995.

23. Claycombe KJ, Jones BH, Standridge MK, Guo Y, Chun JT, Taylor JW and Moustaïd-Moussa N: Insulin increases fatty acid synthase gene transcription in human adipocytes. Am J Physiol 274: 1253-1259, 1998 .

24. Schmid B, Rippmann JF, Tadayyon M and Hamilton BS: Inhibition of fatty acid synthase prevents preadipocyte differentiation. Biochem Biophys Res Commun 328: 1073-1082, 2005.

25. Haunerland NH and Spener F: Fatty acid-binding proteins-insights from genetic manipulations. Prog Lipid Res 43: 328-349, 2004.

26. Chirala SS, Jayakumar A, Gu ZW and Wakil SJ: Human fatty acid synthase: role of interdomain in the formation of catalytically active synthase dimer. Proc Natl Acad Sci USA 98: 3104-3108, 2001.

27. Gonzales AM and Orlando RA: Role of adipocyte-derived lipoprotein lipase in adipocyte hypertrophy. Nutr Metab (Lond) 4: 22, 2007.

28. He Y, Li Y, Zhao T, Wang Y and Sun C: Ursolic acid inhibits adipogenesis in 3T3-L1 adipocytes through LKB1/AMPK pathway. PLoS One 8: e70135, 2013.

29. Zhang XH, Huang B, Choi SK and See JS: Anti-obesity effects of resveratrol-amplified grape skin extracts on 3T3-L1 adipocytes differentiation. Nutr Res Pract 6: 286-293, 2012.

30. Li J, Takaishi K, Cook W, McCorkle SK and Unger RH: Insig-1 'brakes' lipogenesis in adipocytes and inhibits differentiation of preadipocytes. Proc Natl Acad Sci USA 100: 9476-9481, 2003. 